\title{
Hyperspectral Measurements for Estimating Vertical Infection of Yellow Rust on Winter Wheat Plant
}

\author{
Lin-Sheng Huang ${ }^{1}$, Shu-Cun Ju${ }^{1}$, Jin-Ling Zhao ${ }^{1 *}$, Dong-Yan Zhang ${ }^{1}$, Qi-Hong ${ }^{1}$, Ling Teng ${ }^{1}$, Fan Yang ${ }^{1}$ and Yan-Zuo $^{1}$ \\ ${ }^{1}$ Key Laboratory of Intelligent Computing \& Signal Processing, Ministry of Education, Anhui University, China \\ ${ }^{2}$ Anhui General Rural Economic Information Center, China \\ *For correspondence: aling0123@163.com
}

\begin{abstract}
Yellow rust (Puccinia striiformis f. sp. tritici) on winter wheat (Triticum aestivum L.) has resulted in significant reductions in the yield losses and wheat grain quality. It is extremely important to quantitatively detect and assess such a serious disease rather than visual qualitative description. In comparison with traditional diagnosis method, remote sensing has proven to be a cost-effective tool to achieve such a goal. In this study, we used yellow rust in winter wheat to illustrate the capability of estimating the infection index in different leaf layers of the plant using hyperspectral measurements on individual wheat diseased leaves. The analysis results indicated that the severities showed a gradual increasing trend from $\mathrm{F}-1$ ( $\mathrm{F}=\mathrm{Flag}$ leaf) to F-3, while the relative chlorophyll and nitrogen showed an inverse change. The spectral reflectance gradually increased from F-1 to F-3 in the visible and short-wave infrared (SWIR) regions, while it was the very reverse in the near-infrared (NIR) region. In addition, an integral spectral index - yellow rust spectral index (YRSI) was constructed to quantitatively estimate the disease using the most sensitive bands in the visible $(704 \mathrm{~nm})$, NIR (1423 nm) and SWIR (1926 nm) regions. The coefficient of determination $\left(R^{2}\right)$ reaches 0.88 between the disease severity (DS\%) and YRSI, which shows that the index can be suitable and effective to estimate the infection severity for a wheat plant. (C) 2015 Friends Science Publishers
\end{abstract}

Keywords: Hyperspectral reflectance; Spectral signature; Vertical infection; Winter wheat plant; Yellow rust

\section{Introduction}

Diseases can induce severe economic losses in agricultural industry to farmers through reduced yields and increased costs of pesticides (Sankaran et al., 2010). Wheat is the second most important grain crop in China. Nevertheless, various meteorological and biological disasters are threatening China's wheat production. As one of the most destructive diseases of wheat, yellow rust, caused by Puccinia striiformis f. sp. tritici, can cause severe yield losses when susceptible cultivars are grown and weather conditions are favorable for the disease (Wan et al., 2004). To monitor and assess the disease incidence, scouting methods are traditionally employed by visual inspection of the foliage in the field. Unfortunately, only a limited number of field samples can be examined at any one time. These methods have been shown to be inefficient, labor-intensive and time-consuming. Consequently, new instruments and techniques, especially the nondestructive and noninvasive methods, are being rapidly developed to monitor the wheat plants under disease stress.

The emergence of remote sensing techniques has facilitated the identification and monitoring of plant diseases at various spatial and spectral resolutions (Nilsson, 1995; Nicolas, 2004; Huang et al., 2007; Zhang et al., 2014). In comparison with traditional diagnostic methods that rely on visual observation, remote sensing can provide quantitative assessment and scientific analytics for the infection severity and the spatial and temporal diversity. The remote sensing data can be acquired from distances ranging from satellites to ground-based platforms. Initially, wide-band multispectral remote sensing observations have been widely used in detecting disease damage or predicting yield to obtain detailed spatial information for entire wheat fields (Jin et al., 2014). Franke and Menz (2007) used three QuickBird multispectral remote sensing images to execute a spatio-temporal analysis of the leaf rust infection dynamics. Chen et al. (2007) used Landsat Thematic Mapper imageries to detect the take-all disease in wheat. Zhang et al. (2011) used multispectral environment and disaster reduction small satellite images (HJ-CCD) to detect yellow rust in winter wheat by constructing a spectral knowledge base (SKB). Although multi-spectral data can do provide spatial and spectral features for monitoring such a disease, they still lack the potential of detecting subtle changes in plant physical and biochemical properties.

The multispectral satellite systems measure the reflected solar energy in only a few broad channels or wavelength bands. Conversely, hyperspectral data can acquire overall comprehensive infection features of wheat 
yellow rust due to its finer spectral resolutions and more spectral bands. Zhang et al. (2012) used in-situ hyperspectral data for detecting and discriminating yellow rust disease from nutrient stresses. Liu et al. (2014) proposed a new spectral ratio index in the near infrared (NIR) shoulder region (NSRI), defined by a simple ratio of reflectance at 890 $\mathrm{nm}$ to reflectance at $780 \mathrm{~nm}$, for assessing leaf structure deterioration. Ashourloo et al. (2014) developed spectral disease indices for the detection of leaf rust using a spectroradiometer. Zhao et al. (2014) carried out hyperspectral measurements of severity of stripe rust on individual wheat leaves at the heading stage and grain filling stage.

Hyperspectral reflectance data, which can provide a rapid, noninvasive way to detect subtle changes in disease effects, have been intensively employed in investigating the foliar fungal epidemics on both leaf and canopy levels through hundreds of narrow wavelengths. In this study, the in situ hyeperspectral reflectance of diseased wheat leaves in different leaf layers were measured by a hyperspectral field spectrometer. The objective of this work was to estimate the vertical infection of leaves in different leaf layers using hyperspectral reflectance data and some pigment indicators.

\section{Materials and Methods}

\section{Experimental Setup}

To form infected and non-infected leaves, wheat plants were artificially inoculated under controlled conditions in different intensities with yellow rust spores. The wheat cultivar Jing-9428 susceptible to yellow rust was used for the experiment in spring 2011. A spraying method was used here to introduce virulence races of $P$. striiformis f. sp. tritici into living plants. In the laboratory, yellow rust spores were first mixed with distilled water in two sprayers to generate two severity levels (serious and light). The experiment was conducted in the wheat field around 05: $00 \mathrm{pm} .6 .0 \mathrm{~L}$ water was firstly sprayed onto the wheat leaves to make stripe rust spores to easily adhere to the leaves. Then, the mixed suspensions were sprayed on the wheat canopies and a plastic membrane was used to cover the inoculated plots. The following day, the plastic membrane was removed around 08: $30 \mathrm{am}$.

\section{Data Collection}

In compassion with other stages, severe disease had completely colonized the lower leaves at the grain filling stage on May 21. Consequently, the wheat plants at this stage were chosen to estimate the vertical infection induced by yellow rust. The data collection was performed by several portable devices (Fig. 1). To obtain the representative samples, wheat plants were randomly collected from the inoculated and normal fields. F-1, F-2, $\mathrm{F}-3$ ( $\mathrm{F}=$ flag leaf) were obtained from the selected wheat plants (Fig. 1A).

\section{Measurements of Chlorophyll and Nitrogen Content}

Chlorophyll and nitrogen content are two important indicator parameters to demonstrate the growth and nutrient status, both of which are also the important variable for agricultural remote sensing. Two portable meters (SPAD502 and Dualex 4) were used to quickly collect the chlorophyll and nitrogen content of infected and noninfected wheat leaves (Fig. 1B, C and Table 1). The Minolta chlorophyll meter (model SPAD-502) can be used to make quick and easy measurements of leaf greenness. Dualex 4 can simultaneously measure the calculated indices including CHL (the chlorophyll content of the sample), FLAV (the content of flavonols), and NBI (Nitrogen Balance Index, this is the ratio CHL/FLAV, marker of nitrogen deficiency and protein rate) (http://www.force-a.eu/an/dualex.html). Ten diseased and two healthy wheat plant were randomly selected in the inoculated plots and their chlorophyll and nitrogen content of leaves in the different leaf layers were respectively obtained by replicate measurements from top to bottom.

\section{Measurement of Hyperspectral Reflectance}

The in situ hyperspectral reflectance data of wheat sample leaves were measured with a field-portable ASD FieldSpec Pro FR 2500 spectrometer combining with the ASD's Leaf Clip in the visible and near infrared (VNIR) and short-wave infrared (SWIR) ranges (350-2500 nm) (Fig. 1D and E). The spectrometer has a spectral resolution of $3 \mathrm{~nm}$ and a sampling interval of $1.4 \mathrm{~nm}$ between $350-1000 \mathrm{~nm}$ and 2 and $10 \mathrm{~nm}$ in the range of $1000-2500 \mathrm{~nm}$. Most importantly, ASD's Leaf Clip is a portable and powerful tool for measuring foliar hyperspectral data with its own light source in the field. For each leaf, five replicate measurements were performed from the bottom to top, and the average was used as the final reflectance. To suppress possible difference in illumination, the normalized spectral curves were computed by dividing the mean band reflectance of the curve (Eq. 1) (Yu et al., 1999). In addition, the spectral curves within the wavelength ranges of 1330 to $1450 \mathrm{~nm}, 1770$ to $2000 \mathrm{~nm}$ and 2400 to $2500 \mathrm{~nm}$ were removed due to the strong absorption by water vapor.

$$
\operatorname{Ref}_{i}^{\prime}=\frac{\operatorname{Ref}_{i}}{\frac{1}{n} \sum_{i=1}^{n} \operatorname{Ref} f_{i}}
$$

Where, Ref $_{i}{ }^{\prime}$ is the normalized reflectance for band ${ }_{i}$, $R e f_{i}$ is the original reflectance of the band, $n$ is the total number of bands.

\section{Determination of Infection Severity}

The disease severity (DS\%) for each leaf was visually determined by an experienced plant pathologist according to the percentage (Eq. 2). To help in the understanding of yellow rust disease severity on wheat leaves, 
Table 1: Specifications of SPAD-502 and Dualex 4

\begin{tabular}{|c|c|c|c|c|}
\hline Meter & Measurement objects & Measurement area $\left(\mathrm{mm}^{2}\right)$ & Parameters & Value range \\
\hline SPAD-502 & Plant leaves & $2 \mathrm{~mm} \times 3 \mathrm{~mm}$ & Index of relative chlorophyll content & -9.9 to 199.9 SPAD unit \\
\hline Dualex 4 & Plant leaves & $5 \mathrm{~mm}$ diameter & FLAV, CHL, NBI & $\begin{array}{l}0 \sim 3.0 \\
0 \sim 150.00 \text { (DUALEX unit) } \\
0 \sim 999.00 \text { (DUALEX unit) }\end{array}$ \\
\hline
\end{tabular}

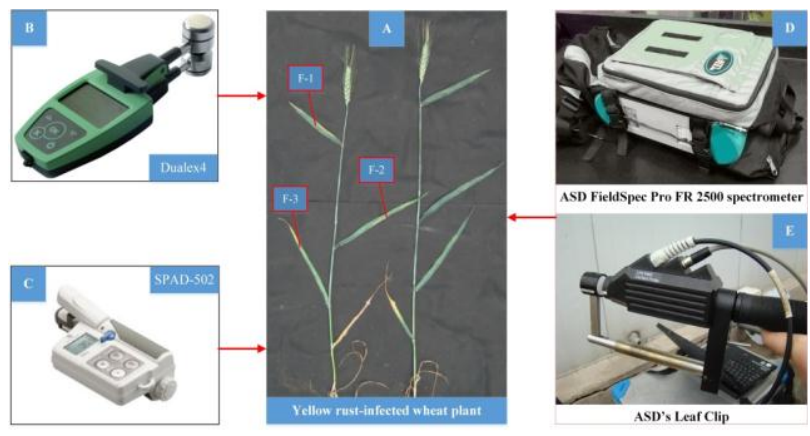

Fig 1: The scheme of data collection using several devices

DISTRAIN (Version 1.0) was used to train the pathologist to estimate disease severity using variegated patterns of disease severity for eight common foliar diseases (including Disease 7-stripe rust) of cereals (Tomerlin and Howell, 1988). The program allows a comparison of estimated severity with actual severity and was developed by The Agricultural Research Service of the U.S. Department of Agriculture Beltsville, Maryland (http://objetoseducacionais2.mec.gov.br/handle/mec/7143).

$$
D S \%=\frac{\sum_{i=1}^{n} S_{i}}{S_{L}} \times 100 \%
$$

Where, $S_{L}$ is the area of a leaf, $i$ is the number of spores and $S_{i}$ is the area of a certain spore on the leaf.

\section{Results}

\section{Infection Characteristics of Leaves at Different Layers}

To investigate the infection differences among different wheat functional leaves, four indicators were used to compare the specific values (Fig. 2). It was obvious that the disease severity generally showed a gradually increasing trend from F-1 to F-3 for the six sample plants (Fig. 2a). This phenomenon indicated that yellow rust infected wheat plant from the lower leaves. Nevertheless, the four indicators did not obviously show the similar trend. The SPAD values showed the trend: $\mathrm{F}-1>\mathrm{F}-2>\mathrm{F}-3$ for the no. 2 , no. 4, no. 6, but they showed the trend: F-1>F-3>F-2 for the no. 1 and no. 5. (Fig. 2b). Similarly, the Chl values had the changing trend (Fig. 2e). The Flav values had the changing trend: F-2>F-1>F-3 except the no. 1 and no. 5 (Fig. 2c). Conversely, the NBI values showed a different trend: F$1>\mathrm{F}-2>\mathrm{F}-3$ except the no. 5 .
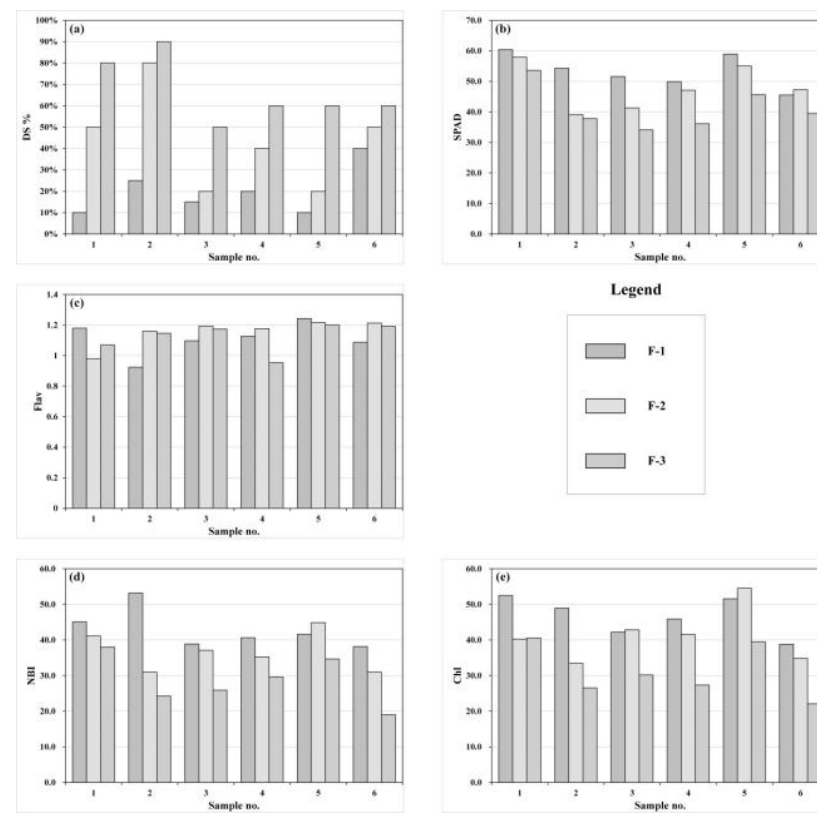

Fig 2: Measured SPAD、Chl、Flav and NBI of leaves in the different leaf layers

It is inevitable that the values measured by the two meters are influenced by some subjective factors (e.g., selected leaf locations, measurement area). To remove a random effect and the individual differences for different wheat plants, the values of four indicators for infectious and non-infectious leaves were averaged (Table 2). In general, the values of four indicators of healthy leaves were greater than that of diseased leaves. Specifically, it was found that the both SPAD and Chl values presented a gradually decreasing trend from F-1 to F-3 for both diseased and healthy leaves, but the corresponding SPAD values were greater than that of Chl. Similarly, the NBI values also showed the changing trend from F-1 to F-3 for diseased leaves, but they presented a gradual increasing trend for healthy leaves. Considering the Flav values, they had the largest values for the F-2 for both diseased and healthy

Table 2: Comparison of SPAD, Chl, Flav and NBI in different leaf layers

\begin{tabular}{lccccc}
\hline Samples & Positions & SPAD & NBI & Chl & Flav \\
\hline \multirow{3}{*}{ Infected leaves } & F-1 & 50.9 & 43.0 & 45.5 & 1.09 \\
& F-2 & 47.5 & 36.6 & 40.1 & 1.13 \\
Healthy leaves & F-3 & 40.8 & 28.8 & 30.1 & 1.09 \\
& F-2 & 61.4 & 48.2 & 56.7 & 1.18 \\
& F-3 & 61.2 & 51.2 & 55.8 & 1.19 \\
\hline
\end{tabular}


leaves, but there was little difference between the three leaves.

\section{Comparison of Spectral Responses for Different Layer Leaves}

To show the spectral differences among the three-layer leaves for an infected wheat plant, their hyperspectral reflectance were acquired (Fig. 3). In general, they have similar spectral response curves for both original reflectance (Fig. 3A) and normalized reflectance (Fig. 3B). It was obvious that two wavelength ranges were useful to differentiate the three leaves in the visible $(520-720 \mathrm{~nm})$, NIR (730-1370 nm) and SWIR (1900-2500 nm) regions. They showed that the reflectance values were gradually increased from F-1 to F-3 in the visible region. The spectral maximum gaps can be used to discriminate the three leaves between the green peak and red valley. However, there was a little difference in the near-infrared region. The reflectance showed a changing trend: $\mathrm{F}-2>\mathrm{F}-1>\mathrm{F}-3$ in the original reflectance. Conversely, it showed that F-1>F-2>F-3 in the near infrared region. There was similar spectral comparison $(\mathrm{F}-1<\mathrm{F}-2<\mathrm{F}-3)$ in the short-wave infrared region just the same as it did in the visible region.

\section{Construction of Yellow Rust Spectral Index}

To describe the overall yellow rust infection for a wheat plant, it is necessary to construct an integral spectral index. Thirty six leaves from thirteen wheat plants were used as the training samples and the correlation analysis was performed between the infection severity and hyperspectral reflectance of leaves at three layers. Consequently, the spectral regions and bands sensitive to yellow rust were found out (Fig. 4A). A composite spectral index (Yellow rust spectral index, YRSI) was constructed in accordance with the different contribution to infection severities of sensitive bands in the visible, NIR and SWIR spectral regions.

$$
\begin{aligned}
& \text { YRSI } \\
& =\frac{R 704_{\text {Healthy }}-R 704_{\text {Diseased }}}{R 704_{\text {Healthy }}} \\
& +\frac{R 1423_{\text {Healthy }}-R 1423_{\text {Diseased }}}{R 1423_{\text {Healthy }}} \\
& +\frac{R 1926_{\text {Healthy }}-R 1926_{\text {Diseased }}}{R 1926_{\text {Healthy }}}
\end{aligned}
$$

Where, $R 704_{\text {Healthy, }} R 1423_{\text {Healthy }}, R 1926_{\text {Healthy }}$ are the spectral reflectance of healthy leaf at $704 \mathrm{~nm}, 1423 \mathrm{~nm}$, $1926 \mathrm{~nm}$, respectively, while $R 704_{\text {Diseased, }} R 1423_{\text {Diseased, }}$, $R 1926_{\text {Diseased }}$ of yellow rust infected leaf at $704 \mathrm{~nm}, 1423 \mathrm{~nm}$, $1926 \mathrm{~nm}$, respectively.

To evaluate the value of such an index, additional thirty leaves from ten plants were used as the testing samples. The linear fitting was performed between the DS\%
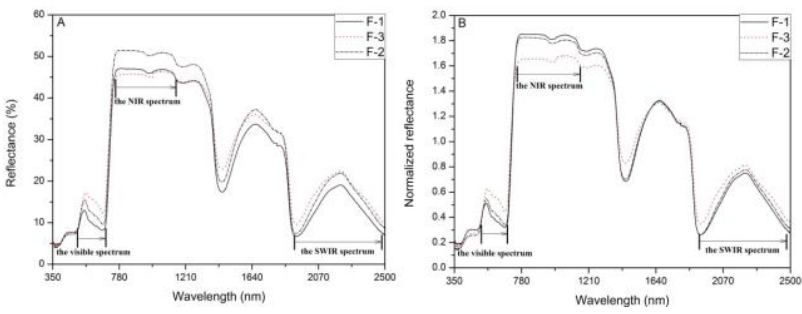

Fig 3: Comparison of spectral responses among different layers
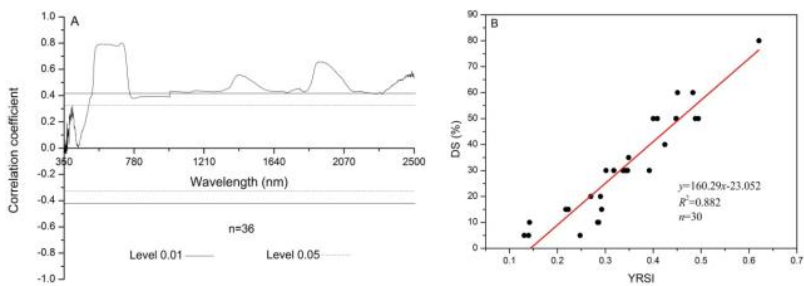

Fig 4: Correlation analysis between the diseased leaves and spectral reflectance
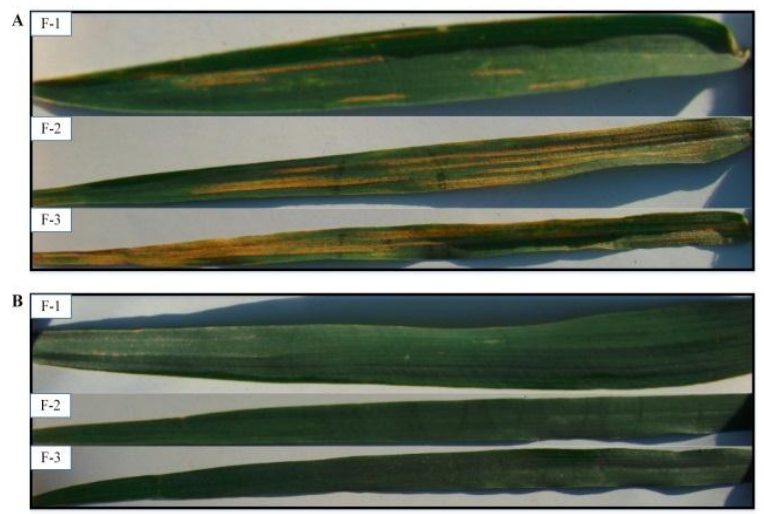

Fig 5: Appearance comparison between infectious and healthy leaves at different layers
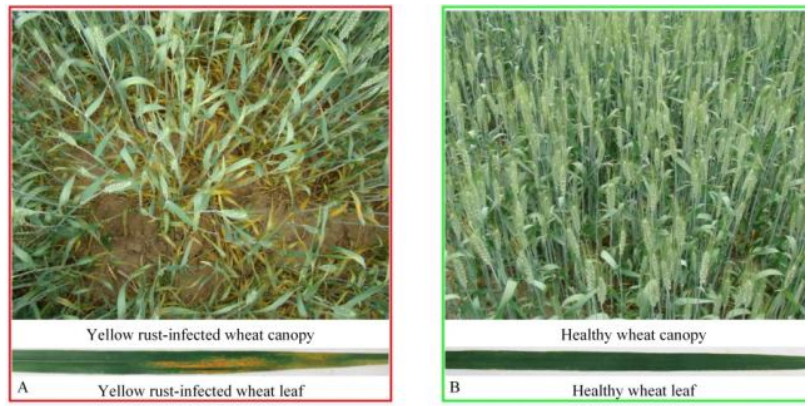

Fig 6: Comparison of yellow rust-infected and healthy wheat canopies and leaves

and YRSI. The coefficient of determination $\left(R^{2}\right)$ reached 0.88 , which showed that this index could be an effective tool to evaluate the infection degree of yellow rust quickly. 


\section{Discussion}

When a wheat plant is infected by yellow rust, it is necessary to quickly acquire some pigment indicators (e.g., chlorophyll, nitrogen) to estimate the severity. In comparison with traditional laboratory-derived tests, a chlorophyll meter can conveniently estimate foliar chlorophyll and nitrogen $(\mathrm{N})$ contents in many species (Liu et al., 2012). SPAD-502 and Dualex 4 are the two commonly used meters, and both SPAD and Chl derived from the two devices can be used to present the relative chlorophyll content. Nevertheless, the values can be affected by the measuring locations of leaves, so it is necessary to get the average value by repeating a measurement several times from top to bottom of a leaf (Fig. 2 and Table 2). In our study, the two meters showed a good consistency (Fig. 2b and 2e).

The yellow rust pathogen resulted in a reduction in total chlorophyll content in all wheat leaves with the increase in severity. As shown in Fig. 5, the severity increased from F-1 to F-3, so the chlorophyll showed also a gradual decreasing trend. Similarly, the NBI showed the same changing trend. The reason is characterized in that nitrogen tends to transfer to vigorous plant organism. An obvious vertical distribution of nitrogen content can be found in different layers of leaf and stem (Wang et al., 2005). The nitrogen content of lower leaves begins to transfer to the upper layer during middle-late growth stages, so it is a unique feature that nitrogen content shows a vertical gradient in the plant canopy (Markus et al., 2003). The F-1 is located in the upper layer of the wheat plant, so it maintained relatively higher photosynthetic capacity.

It is very useful to derive the overall infection from the leaves in different layers for a wheat plant. The leaves at different layers usually show different symptoms when infected by yellow rust pathogen (Chen et al., 2014). In comparison with healthy leaves (Fig. 5A), they are visually covered by a mass of yellow-colored stripes produced parallel along the venations of each leaf blade (Fig. 5B). Actually, the foliar chlorophyll, water and internal leaf structure have been destroyed by the disease. It is extremely important to quickly and quantitatively estimate the potential damage for farmers and agricultural decision makers. Hyperspectral remote sensing has been proved to be an effective tool for detection and differentiation of plant diseases (Moshou et al., 2004; Devadas et al., 2009; Yuan et al., 2014).

In general, the leaf reflectance for green vegetation is controlled by different dominant factors in the visible, NIR and SWIR portions of the electromagnetic spectrum. Leaf pigments, cell structure and water content are respectively the three primary factors for the three wavelength ranges (Huang et al., 2007; Huang et al., 2012). The yellow rust destroys the chlorophyll absorption of infections leaves, so there is a higher reflectance for a high severity compared to a mild severity. The reflectance showed a gradual decreasing trend from F-1 to F-3 in the visible region (Fig. 3). Conversely, there is a spectral decrease from F-3 to F-1 in the NIR spectrum due to the destruction of cell structure at the leaf locations coved with yellow rust pustules (West et al., 2003). There was a little difference for the F-1 and F-2 in the original and normalized spectral reflectance. The reason was that the original reflectance could be influenced by light radiation, so the normalization should be performed to show the correct spectral comparison. In the SWIR spectrum, foliar water content was reduced by the disease and the reflectance showed a gradual decreasing trend from F-1 to F-3. Consequently, a composite index combing the three spectral regions is very essential to describe and estimate the disease severity of yellow rust for a wheat plant.

The leaf is one of important portions for a wheat plant. In general, the leaves are firstly covered by such a disease and visually observed by a farmer. Consequently, wheat leaves at different layers were used as the study objects to identify the vertical infection caused by yellow rust. In previous studies in monitoring wheat diseases using remote sensing, the spectral data are usually acquired on the top of wheat canopy. This approach is useful to estimate the disease when pathogen has infected the top layer leaves (Fig. 6). Actually, the disease has infected the wheat when it is visually observed. When conditions are wet during the season, the yellow rust spores are dispersed by the wind and can result in rapid disease development higher up the plant; and in neighbouring wheat crops (Chakraborty et al., 2011). Therefore, it is important to monitor and estimate the severity, especially on the early infection of seedlings. Our study can explore a case study for multi-angular remote sensing.

\section{Conclusion}

Hyperspectral reflectance and some portable meters are very effective and useful to quantitatively estimate the disease severity caused by yellow rust. In this study, some infection features were identified by spectral reflectance and pigment indicators. Consequently, the disease severities showed a gradual increase from F-1 to F-3. Conversely, the relative chlorophyll and nitrogen contents had a reverse change. With the increase of infection severities, the spectral differences of leaves can be obviously observed over the hyperspectral curves. An integral spectral index (YRSI) derived from the most sensitive bands in the visible, NIR and SWIR regions is necessary and important to estimate the disease severities. The coefficient of determination $\left(R^{2}\right)$ can reach 0.88 between the disease severity (DS\%) and YRSI. Thus, the index is suitable for the determination of disease degree of yellow rust.

\section{Acknowledgements}

The project was supported by National Natural Science Foundation of China (41201422), the Open Research Fund of Key Laboratory of Digital Earth Science, Institute of Remote Sensing and Digital Earth, Chinese Academy of Sciences (No. 2014LDE012), Leadership Introduction 
Project of Academy and Technology of Anhui University (10117700024), 211 Project of Anhui University (KJQN1121) and open project (12KF004) from State Key Laboratory of ASIC \& System, Fudan University.

\section{References}

Ashourloo, D., M.R. Mobasheri and A. Huete, 2014. Developing two spectral disease indices for detection of wheat leaf rust (Puccinia triticina). Remote Sens., 6: 4723-4740

Chakraborty, S., J. Luck, G. Hollaway, G. Fitzgerald and N. White, 2011. Rust-proofing wheat for a changing climate. Euphytica, 179: 19-32

Chen, W., C. Wellings, X. Chen, Z. Kang and T. Liu, 2014. Wheat stripe (yellow) rust caused by Puccinia striiformis f. sp. tritici. Mol. Plant Pathol., 15: 433-46

Chen, X., J. Ma, H. Qiao, D. Cheng, Y. Xu and Y. Zhao, 2007. Detecting infestation of take-all disease in wheat using Landsat Thematic Mapper imagery. Int. J. Remote Sens., 28: 5183-5189

Devadas, R., D.W. Lamb, S. Simpfendorfer and D. Backhouse, 2009. Evaluating ten spectral vegetation indices for identifying rust infection in individual wheat leaves. Precis. Agric., 10: 459-470

Franke, J. and G. Menz, 2007. Multi-temporal wheat disease detection by multi-spectral remote sensing. Precis. Agric., 8: 161-172

Huang, L., J. Zhao, D. Zhang, L. Yuan, Y. Dong and J. Zhang, 2012. Identifying and mapping stripe rust in winter wheat using multitemporalairbornehyperspectralimages.Int.J.Agric. Biol., 14: 697-704

Huang, W., D.W. Lamb, Z. Niu, Y. Zhang, L. Liu and J. Wang, 2007. Identification of yellow rust in wheat using in-situ spectral reflectance measurements and airborne hyperspectral imaging. Precis. Agric., 8: 187-197

Jin, X.L., X.G. Xu, H.K. Feng, X.Y. Song, Q. Wang, J.H. Wang, W.S. Guo, 2014. Estimation of grain protein content in winter wheat by using three methods with hyperspectral data. Int. J. Agric. Biol., 16: 498-504

Liu Z.A., J.P. Yang and Z.C. Yang, 2012. Using a chlorophyll meter to estimate tea leaf chlorophyll and nitrogen contents. J. Soil Sci. Plant Nutr., 12: 339-348

Liu, L.Y., W.J. Huang, R.L. Pu and J.H. Wang, 2014. Detection of internal leaf structure deterioration using a new spectral ratio index in the near-infrared shoulder region. J. Integr. Agric., 13: 760-769

Markus, L., 2003. Vertical leaf nitrogen distribution in relation to nitrogen status in grassland plants. Ann. Bot., 92: 679-688

Moshou, D., C. Bravo, J. West, S. Wahlen, A. McCartney and H. Ramon, 2004. Automatic detection of 'yellow rust' in wheat using reflectance measurements and neural networks. Comput. Electron. Agric., 44: $173-188$
Nicolas, H., 2004. Using remote sensing to determine of the date of a fungicide application on winter wheat. Crop Prot., 23: 853-863

Nilsson, H.E., 1995. Remote sensing and image analysis in plant pathology. Can. J. Plant Pathol., 17: 154-166

Sankaran, S., A. Mishra, R. Ehsani and C. Davis, 2010. A review of advanced techniques for detecting plant diseases. Comput. Electron. Agric., 72: 1-13

Tomerlin, J.R. and A. Howell, 1988. ADISTRAIN: a computer program for training people to estimate disease severity on cereal leaves. Plant Dis., 72: 455-459

Wan, A., Z. Zhao, X. Chen, Z. He, S. Jin, Q. Jia, G. Yao, J. Yang, B. Wang, G. Li, Y. Bi and Z. Yuan, 2004. Wheat stripe rust epidemic and virulence of Puccinia striiformis $\mathrm{f}$. sp. tritici in China in 2002. Plant Dis., 88: 896-904

Wang, Z., J. Wang, C. Zhao, M. Zhao, W. Huang and C. Wang, 2005. Vertical distribution of nitrogen in different layers of leaf and stem and their relationship with grain quality of winter wheat. J. Plant Nutr., 28: 73-91

West, J.S., C. Bravo, R. Oberti, D. Lemaire, D. Moshou and H.A. McCartney, 2003. The potential of optical canopy measurement for targeted control of field crop diseases. Annu. Rev. Phytopathol., 41: 593-614

Yu, B., M. Ostland, P. Gong and R. Pu, 1999. Penalized discriminant analysis of in situ hyperspectral data for conifer species recognition IEEE Trans. Geosci. Remote Sens., 37: 2569-2577

Yuan, L., Y. Huang, R.W. Loraamm, C. Nie, J. Wang and J. Zhang, 2014. Spectral analysis of winter wheat leaves for detection and differentiation of diseases and insects. Field Crops Res., 156: 199207

Zhang, J., R. Pu, R.W. Loraamm G. Yang and J. Wang, 2014. Comparison between wavelet spectral features and conventional spectral features in detecting yellow rust for winter wheat. Comput. Electron. Agric., 100: 79-87

Zhang, J., R. Pu, W. Huang, L. Yuan, J. Luo and J. Wang, 2012. Using insitu hyperspectral data for detecting and discriminating yellow rust disease from nutrient stresses. Field Crops Res., 134: 165-174

Zhang, J., W. Huang, J. Li, G. Yang, J. Luo, X. Gu and J. Wang, 2011. Development, evaluation and application of a spectral knowledge base to detect yellow rust in winter wheat. Precis. Agric., 12: 716731

Zhao, J., L. Huang, W. Huang, D. Zhang, L. Yuan, J. Zhang and D. Liang, 2014. Hyperspectral measurements of severity of stripe rust on individual wheat leaves. Eur. J. Plant Pathol., 139: 401-411

(Received 09 March 2015; Accepted 20 April 2015) 